\title{
EVALUACIÓN DE UNA INTERVENCIÓN PARA EL USO DE TROMBOPROFILAXIS BASADA EN EL ENVÍO DE ALERTAS ELECTRÓNICAS A MÉDICOS RESIDENTES DE PRIMERAÑO DE DOS HOSPITALES DE LIMA, PERÚ
}

\author{
Roberto Delgado-Zapata1,a, Ray Ticse ${ }^{1,2, b, c}$, María de los Ángeles Gamarra1,a, Katherine Gómez,a, \\ Wilfredo Mormontoy ${ }^{3, d}$
}

\begin{abstract}
RESUMEN
Objetivos. Evaluar la aplicación de una intervención basada en el envío de mensajes de texto y correos electrónicos dirigido a médicos residentes de medicina interna para lograr el incremento de la prescripción de tromboprofilaxis acorde a Guías de Práctica Clínica (GPC). Materiales y métodos. Se realizó un estudio tipo cuasiexperimental en los servicios de hospitalización de medicina interna de dos hospitales generales de Lima, Perú, uno seleccionado para aplicar la intervención y el otro como control. La unidad de análisis fue la hoja de prescripción médica. Se consideró que la intervención debería incrementar la prescripción acorde a GPC con respecto a la evaluación inicial y también respecto al hospital control. Resultados. Se evaluaron 394 hojas de prescripción médica, 227 (57,4\%) procedentes del servicio intervenido y $167(42,4 \%)$ correspondieron del control. En el servicio intervenido, inicialmente el 64,5\% tuvo una prescripción acorde a GPC, para disminuir a 54,4\% luego de la intervención; el sobreuso aumentó de 13,6 a $26,8 \%$. Conclusiones. La intervención basada en el envío de mensajes de texto y correos electrónicos no tuvo impacto, observándose una tendencia al sobreuso de tromboprofilaxis posintervención.
\end{abstract}

Palabras clave: tromboprofilaxis; Mensaje de texto; Mensajes electrónicos, Guías de práctica clínica; Tecnología de la información; Educación médica (Fuente: DeCS BIREME).

\section{EVALUATION OF AN INTERVENTION FOR THE USE OF THROMBOPROPHYLAXIS BASED ON ELECTRONIC ALERT TRANSMISSION TO FIRST-YEAR MEDICAL RESIDENTS FROM TWO HOSPITALS OF LIMA, PERU}

\begin{abstract}
Objectives. To assess the implementation of an intervention based on the sending of text messages and e-mails to internal medicine residents in order to achieve an increase in the prescription of thromboprophylaxis according to Clinical Practice Guidelines (CPG). Materials and methods. A quasi-experimental study was conducted in the inpatient units of internal medicine at two general hospitals in Lima, Peru: one selected to implement the intervention and the other as control. The unit of analysis was the therapeutic order sheet. It was considered that the intervention should increase the order of thromboprophylaxis according to CPG with respect to the initial assessment and also in relation to the control hospital. Results. A total of 394 therapeutic order sheets, 227 (57.4\%) from the intervention hospital and $167(42.4 \%)$ from the control, were evaluated. In the intervention hospital, $64.5 \%$ initially had an order of thromboprophylaxis according to CPG, which decreased to $54.4 \%$ after the intervention; overuse increased from 13.6 to $26.8 \%$. Conclusions. Intervention based on sending text messages and e-mails had no impact, showing a tendency to overuse of thromboprophylaxis post-intervention.
\end{abstract}

Key words: Thromboprophylaxis; Text messaging; Electronic mail; Practice guidelines; Information technology; Medical education (Source: MeSH NLM).

\section{INTRODUCCIÓN}

El tromboembolismo venoso (TEV) es una condición frecuente en pacientes hospitalizados, siendo sus manifestaciones más comunes la trombosis venosa profunda (TVP) y el tromboembolismo pulmonar (TEP), que se asocia con eventos que aumentan el tiempo de estancia hospitalaria, costos y morbimortalidad del paciente ${ }^{(1)}$. Se ha evidenciado que la mayoría de estos pacientes tienen uno o más factores de riesgo que los predispone a estas condiciones ${ }^{(2)}$.

Facultad de Medicina, Universidad Peruana Cayetano Heredia. Lima, Perú.

Servicio de Endocrinología. Hospital Nacional Cayetano Heredia. Lima, Perú.

Departamento de Estadística, Demografía, Humanidades y Ciencias Sociales. Facultad de Ciencias, Universidad Peruana Cayetano Heredia. Lima, Perú

Estudiante de Medicina; ${ }^{\mathrm{b}}$ Médico especialista en Medicina Interna y Endocrinología; ${ }^{\mathrm{c}}$ Maestro en Epidemiología Clínica; ${ }^{\mathrm{d}}$ Maestro en Salud Pública.

Recibido: 11/03/2015 Aprobado: 09/03/2016

Citar como: Delgado-Zapata R, Ticse R, Gamarra MdlA, Gómez K, Mormontoy W. Evaluación de una intervención para el uso de tromboprofilaxis basada en el envío de alertas electrónicas a médicos residentes de primer año de dos hospitales de Lima, Perú. Rev Peru Med Exp Salud Publica. 2016;33(3):498-506. doi:10.17843/rpmesp.2016.333.2372 
EI TEP es la causa prevenible más frecuente de muerte hospitalaria y su prevención constituye la principal estrategia para mejorar la seguridad de los pacientes hospitalizados ${ }^{(3)}$. Si bien no se conocen cifras exactas sobrelaincidencia de TEP, se ha calculado quela incidencia anual es de 150 a 200 casos por 100000 habitantes ${ }^{(4)}$. Existen numerosos estudios que demuestran que el uso rutinario en salas de hospitalización de tromboprofilaxis reduce los episodios de TVP y TEP, así como los costos generales de la hospitalización ${ }^{(5-7)}$.

Se han desarrollado guías de práctica clínica (GPC) para la prescripción de tromboprofilaxis basada en la estratificación del riesgo para TEP o TVP ${ }^{(2,8-11)}$. Sin embargo, se reporta que solo el $40 \%$ de la población adulta hospitalizada que cursa con alto riesgo de TVP recibe tromboprofilaxis ${ }^{(12)}$. Muchas veces se les indica tromboprofilaxis, pero a dosis inadecuadas ${ }^{(13)}$.

Se recomiendan intervenciones activas y evaluaciones periódicas para incrementar la aplicación de tromboprofilaxis ${ }^{(14,15)}$. Se han aplicado tecnologías de información y comunicación (TIC), las cuales son herramientas computacionales e informáticas que procesan, almacenan, sintetizan y representan la información de la forma más variada. Algunos ejemplos son los dispositivos móviles, los blogs, la web y los wikis ${ }^{(16)}$. Las TIC están siendo implementados con éxito en diversas áreas de la medicina, cumpliendo un rol importante en el aprendizaje de los médicos, en la relación médico-paciente, en la adherencia al tratamiento y en la prescripción de fármacos ${ }^{(17-19)}$. El objetivo principal del estudio es evaluar el impacto de una intervención basada en el envío de mensajes de texto a celulares y correos electrónicos sobre el uso de tromboprofilaxis a médicos residentes en un servicio de medicina.

\section{MATERIALES Y MÉTODOS}

\section{DISEÑO Y POBLACIÓN DE ESTUDIO}

Se realizó un estudio tipo cuasiexperimental con grupo control, en el que se evaluó la prescripción de tromboprofilaxis en las salas de hospitalización de medicina interna en dos hospitales generales de Lima Perú, Hospital Cayetano Heredia $(\mathrm{HCH})$ y Hospital Arzobispo Loayza (HAL), luego de lo cual se realizó una intervención basado en el envío de alertas electrónicas sobre la prescripción acorde a las recomendaciones de las GPC en uno de los hospitales mencionados. se utilizó el Score de Padua para calcular el riesgo de TEV y la Guía de Práctica Clínica Basada en Evidencia sobre Prevención de TEV en Pacientes No Quirúrgicos del American College of Chest Physicians (ACCP) - 9a Edición (2).

Para cada medición, se tomó como unidad de análisis la hoja de terapéutica de cada uno de los pacientes hospitalizados en los servicios de Medicina de los hospitales mencionados. Se incluyeron todas las hojas de terapéutica en las cuales se podía identificar al paciente mediante el nombre, número de cama o historia clínica. Además, debían de tener las fechas de cuándo los fármacos habían sido prescritos, y que figure la firma y el sello del médico asistente y médico residente de primer año a cargo. Por otro lado, se excluyeron aquellas hojas de terapéutica en las cuáles no se tuvo acceso a la historia clínica, el paciente tenía indicación de terapia antitrombótica, o que al momento del estudio estuviera recibiendo tromboprofilaxis crónica.

La hipótesis que planteamos fue que una intervención basada en alertas electrónicas incrementaría la prescripción de tromboprofilaxis por médicos residentes. El cálculo del tamaño de la muestra para cada medición en cada uno de los hospitales se realizó mediante una diferencia de proporciones. Se incluyó dentro del cálculo, la comparación del impacto basal y posintervención, obteniéndose que por cada medición se necesitaba un mínimo de 59 hojas de terapéutica prescritas por cada hospital participante. Para esta intervención se esperó incrementar la prescripción adecuada de 70 a 90\%. Se tomó el valor de $70 \%$ como referencia de prescripción acorde a GPC de tromboprofilaxis, basado en una evaluación previa realizada en estos hospitales. Consideramos que es clínicamente relevante un aumento del $20 \%$ de la frecuencia de prescripción de tromboprofilaxis. Como hipótesis secundaria planteamos que en el servicio de medicina interna del hospital intervenido la prescripción acorde a GPC sería superior al del hospital control, esto porque algún incremento podría deberse no sólo a la aplicación de intervención sino a la adquisición de mayor destreza clínica durante este periodo.

\section{DEFINICIÓN DE VARIABLES}

Se trabajó en dos hospitales generales de Lima, Perú en los cuáles en uno de ellos se realizó la intervención propuesta y el otro se utilizó como control; es decir, no se intervino. Durante cada medición se clasificó a los pacientes incluidos como "paciente con indicación de tromboprofilaxis", si sus factores de riesgo sumaban un puntaje $\geq$ a 4 según lo establecido por el score de Padua y la guía de la ACCP, y por lo cual debían recibir tromboprofilaxis ${ }^{(2,20)}$. La tromboprofilaxis farmacológica recomendada fue heparina sódica (HNF) 5000 UI SC 2v/d o $3 \mathrm{v} / \mathrm{d}$, o enoxaparina $40 \mathrm{mg}$ SC 1v/d. Aquellos pacientes que no podían recibir tromboprofilaxis farmacológica dado que presentaba un alto riesgo de sangrado como úlcera gastroduodenal activa, sangrado en los 3 meses previos o plaquetas < 50000 unidades $/ \mathrm{mm}^{3}$, se clasificaron como "paciente con contraindicación de tromboprofilaxis farmacológica". Para estos últimos la recomendación su el uso de tromboprofilaxis mecánica como medias antitrombóticas o compresión neumática intermitente.

Luego, se revisó la hoja de terapéutica y se definió si cada una de ella tenía una "prescripción acorde a GPC". Se consideró dentro de esta variable a todas aquellas prescripciónes de tromboprofilaxis en un paciente con indicación y dosis acorde a lo indicado en la GPC. Además, se incluyó dentro de esta variable a la ausencia de prescripción en un paciente que no tenía indicación de tromboprofilaxis. Se definió como "subuso" a los pacientes con indicación, pero sin prescripción de tromboprofilaxis. Al contrario, aquellos pacientes con 
prescripción y sin indicación de tromboprofilaxis, se le llamó "sobreuso" (Anexo 1).

\section{PROCEDIMIENTOS Y TÉCNICAS}

Se trabajó en las salas de hospitalización de servicios de Medicina Interna del HCH y el HAL. Para este estudio, se revisaron las hojas de prescripción médica y se evaluó la variación de la prescripción de tromboprofilaxis durante 21 semanas, se realizaron 3 mediciones. Estas mediciones de la frecuencia de prescripción se realizaron al inicio del estudio y, en las primeras 72 horas luego de finalizar los 2 y 4 meses del inicio de la intervención. Las hojas de terapéutica revisadas correspondieron al día de la evaluación y cinco días previos a la misma.

\section{INTERVENCIÓN}

De los dos hospitales en los que se trabajó, se decidió de manera aleatoria el hospital a realizar la intervención dado que no se encontraron diferencias significativas en la medición basal al inicio del estudio. Previo al inicio de la intervención se realizó una reunión con los médicos residentes en cada uno de los hospitales participantes, donde se les expuso los resultados hallados en la medición basal. Además, Se les entregó una cartilla informativa en la que se resumían los factores de riesgo para desarrollar TVP/TEP según el score de Padua, y las dosis de tromboprofilaxis según la guía de la ACCP. Antes de comenzar la intervención en el hospital elegido, se realizó un grupo focal con los médicos residentes de primer año para saber el conocimiento sobre tromboprofilaxis y su subuso, así como desarrollar el contenido de los mensajes de texto y elegir aquellos con una respuesta más positiva. Los mensajes seleccionados fueron distribuidos a todos los médicos residentes del servicio a intervenir durante el periodo de estudio. Se inició 4 semanas después de la medición basal hasta la semana 21, siendo enviados dos veces por semana. El contenido de los mensajes de texto fueron casos modelo de pacientes con riesgo de TEV, con indicación de recibir tromboprofilaxis 0 deambulación precoz. La lista de mensajes enviados se detalla en el Anexo 2. Asimismo, se les envió un correo electrónico una vez al mes con los datos de la cartilla informativa tras la medición basal: desde la semana 4 hasta la semana 21 del estudio.

\section{ANÁLISIS ESTADÍSTICO}

Se calculó la media aritmética y desviación estándar, o mediana y rango intercuartílico para las variables cuantitativas según hayan tenido distribución normal o no, y proporciones para variables cualitativas. Se aplicó el test de chi cuadrado para comparar los grupos respecto a variables cualitativas. En este caso, el nivel de significación asumido fue $\alpha=0,05$. Para el procesamiento y análisis se utilizó STATA v.12.

\section{CONSIDERACIONES ÉTICAS}

Este protocolo y el consentimiento informado fueron evaluados y aprobados por el Comité Institucional de Ética de la Universidad Peruana Cayetano Heredia, el Hospital Nacional Arzobispo Loayza y el Hospital Nacional Cayetano Heredia. Además, todos los participantes en el estudio firmaron un consentimiento informado en el cuál se les expuso los objetivos del estudio, los procedimientos a realizar, los riesgos y beneficios.

\section{RESULTADOS}

Se recolectaron 415 hojas de terapéutica correspondientes al mismo número de pacientes. Se excluyeron las hojas de terapéutica correspondiente a 21 pacientes: diez por diagnóstico actual de TVP, cinco por TEP, tres por recibir anticoagulación crónica profiláctica y tres por no tener hojas de terapéutica con sello de asistente al momento del estudio. De los 394 hojas de terapéutica restantes, 42,4\% ( $n=167)$ procedieron del hospital utilizado como control y $57,6 \%$ $(n=227)$ del hospital intervenido.

La media y desviación estándar $( \pm \mathrm{DE})$ de la edad de los pacientes fue $56,6 \pm 20,1$ años y el $68,3 \%(n=269)$ de ellos fueron mujeres, siendo este el sexo predominante en las tres mediciones. Las características generales de la población se detallan en la Tabla 1.

Tabla 1. Características generales de los pacientes hospitalizados en los servicios de medicina de dos hospitales generales de Lima

\begin{tabular}{|c|c|c|c|c|}
\hline Características & $\begin{array}{l}\text { Medición basal } \\
(n=122)\end{array}$ & $\begin{array}{l}\text { Primera medición } \\
\qquad(n=130)\end{array}$ & $\begin{array}{c}\text { Segunda medición } \\
(n=142)\end{array}$ & $\begin{array}{c}\text { Total } \\
(n=394)\end{array}$ \\
\hline \multicolumn{5}{|l|}{ Hospital, n (\%) } \\
\hline Control & $60(49,2 \%)$ & $55(42,3 \%)$ & $52(36,6 \%)$ & $167(42,4 \%)$ \\
\hline Intervenido & $62(50,8 \%)$ & $75(57,7 \%)$ & $90(63,4 \%)$ & $227(57,6 \%)$ \\
\hline \multicolumn{5}{|l|}{ Sexo, n (\%) } \\
\hline Femenino & $83(68,0 \%)$ & $92(70,8 \%)$ & $94(66,2 \%)$ & $269(68,3 \%)$ \\
\hline Masculino & $39(31,9 \%)$ & $38(29,2 \%)$ & $48(33,8 \%)$ & $125(31,7 \%)$ \\
\hline \multicolumn{5}{|l|}{ Edad, n (\%) } \\
\hline Media & $60,1 \pm 20,2$ & $53,5 \pm 20,1$ & $56,5 \pm 19,6$ & $56,6 \pm 20,1$ \\
\hline Menor de 70 años & $78(63,9 \%)$ & $103(79,2 \%)$ & $102(71,8 \%)$ & $283(71,8 \%)$ \\
\hline Mayor o igual a 70 años & $44(36,1 \%)$ & $27(20,8 \%)$ & $40(28,2 \%)$ & $111(28,2 \%)$ \\
\hline
\end{tabular}


Tabla 2. Frecuencia de pacientes con indicación y contraindicación de tromboprofilaxis hospitalizados en los servicios de medicina de dos hospitales generales de Lima

\begin{tabular}{|c|c|c|c|c|c|c|c|c|c|c|c|c|c|}
\hline \multirow{2}{*}{ Características } & \multicolumn{3}{|c|}{ Medición basal } & \multirow{2}{*}{$\begin{array}{c}\text { Valor } \\
p\end{array}$} & \multicolumn{3}{|c|}{ Primera medición } & \multirow{2}{*}{$\begin{array}{c}\text { - Valor } \\
p\end{array}$} & \multicolumn{3}{|c|}{ Segunda medición } & \multirow{2}{*}{$\begin{array}{c}\text { Valor } \\
p\end{array}$} & \multirow{2}{*}{$\begin{array}{c}\text { Total } \\
(n=394)\end{array}$} \\
\hline & $\begin{array}{c}\text { Total } \\
(n=122)\end{array}$ & $\begin{array}{c}\text { Control } \\
(n=60)\end{array}$ & $\begin{array}{l}\text { Intervenido } \\
\quad(n=62)\end{array}$ & & $\begin{array}{c}\text { Total } \\
(n=130)\end{array}$ & $\begin{array}{c}\text { Control } \\
(n=55)\end{array}$ & $\begin{array}{l}\text { Intervenido } \\
\quad(n=75)\end{array}$ & & $\begin{array}{c}\text { Total } \\
(n=142)\end{array}$ & $\begin{array}{c}\text { Control } \\
(n=52)\end{array}$ & $\begin{array}{l}\text { Intervenido } \\
\qquad(n=90)\end{array}$ & & \\
\hline \multicolumn{14}{|c|}{$\begin{array}{l}\text { Indicación de } \\
\text { Tromboprofilaxis, } \\
\text { n (\%) }\end{array}$} \\
\hline Sí & $\begin{array}{c}55 \\
(45,1 \%)\end{array}$ & $\begin{array}{c}32 \\
(53,3 \%)\end{array}$ & $\begin{array}{c}23 \\
(37,1 \%)\end{array}$ & 0,072 & $\begin{array}{c}53 \\
(40,8 \%)\end{array}$ & $\begin{array}{c}24 \\
(43,6 \%)\end{array}$ & $\begin{array}{c}176 \\
(44,7 \%)\end{array}$ & 0,569 & $\begin{array}{c}68 \\
(47,9 \%)\end{array}$ & $\begin{array}{c}33 \\
(63,5 \%)\end{array}$ & $\begin{array}{c}35 \\
(38,9 \%)\end{array}$ & 0,005 & $\begin{array}{c}176 \\
(44,7 \%)\end{array}$ \\
\hline No & $\begin{array}{c}67 \\
(54,9 \%)\end{array}$ & $\begin{array}{c}28 \\
(46,7 \%)\end{array}$ & $\begin{array}{c}39 \\
(62,9 \%)\end{array}$ & & $\begin{array}{c}77 \\
(59,2 \%)\end{array}$ & $\begin{array}{c}31 \\
(56,4 \%)\end{array}$ & $\begin{array}{c}218 \\
(55,3 \%)\end{array}$ & & $\begin{array}{c}74 \\
(52,1 \%)\end{array}$ & $\begin{array}{c}19 \\
(36,5 \%)\end{array}$ & $\begin{array}{c}55 \\
(61,1 \%)\end{array}$ & & $\begin{array}{c}218 \\
(55,3 \%)\end{array}$ \\
\hline \multicolumn{14}{|c|}{$\begin{array}{l}\text { Contraindicación } \\
\text { de tromboprofilaxis } \\
\text { farmacológica, } n(\%)\end{array}$} \\
\hline Sí & $\begin{array}{c}16 \\
(13,1 \%)\end{array}$ & $\begin{array}{c}7 \\
(11,7 \%)\end{array}$ & $\begin{array}{c}9 \\
(14,5 \%)\end{array}$ & 0,641 & $\begin{array}{c}12 \\
(9,2 \%)\end{array}$ & $\begin{array}{c}7 \\
(12,7 \%)\end{array}$ & $\begin{array}{c}57 \\
(14,5 \%)\end{array}$ & 0,238 & $\begin{array}{c}29 \\
(20,4 \%)\end{array}$ & $\begin{array}{c}17 \\
(32,7 \%)\end{array}$ & $\begin{array}{c}12 \\
(13,3 \%)\end{array}$ & 0,006 & $\begin{array}{c}57 \\
(14,5 \%)\end{array}$ \\
\hline No & $\begin{array}{c}106 \\
(86,9 \%)\end{array}$ & $\begin{array}{c}53 \\
(88,3 \%)\end{array}$ & $\begin{array}{c}53 \\
(85,5 \%)\end{array}$ & & $\begin{array}{c}118 \\
(90,8 \%)\end{array}$ & $\begin{array}{c}48 \\
(87,3 \%)\end{array}$ & $\begin{array}{c}337 \\
(85,5 \%)\end{array}$ & & $\begin{array}{c}113 \\
(79,6 \%)\end{array}$ & $\begin{array}{c}35 \\
(67,3 \%)\end{array}$ & $\begin{array}{c}78 \\
(86,7 \%)\end{array}$ & & $\begin{array}{c}337 \\
(85,5 \%)\end{array}$ \\
\hline
\end{tabular}

Del total de hojas de terapéutica de los pacientes evaluados, el 44,7\% $(n=176)$ tenían indicación de tromboprofilaxis, siendo dentro de este grupo el factor de riesgo más frecuente en ambos hospitales la movilización reducida a lo largo de las tres mediciones: 90,9\% $(n=50), 77,4 \%(n=41)$ y $85,3 \%(n=58)$, respectivamente. Mientras que la edad $\geq$ a 70 años e infección aguda y/o enfermedad reumatológica fueron los otros factores de riesgo con más frecuencia. Por otra parte, el total de pacientes que presentaron alguna contraindicación para recibir tromboprofilaxis farmacológica fue de $14,5 \%$ $(n=57)$ (Tabla 2 y 3$)$.

En el hospital intervenido, la prescripción acorde a GPC en la medición basal fue de $64,5 \%(n=40)$; en la primera medición de $62,7 \%(n=47)$, y en la segunda de $54,4 \%$

Tabla 3. Factores de riesgo para el desarrollo de tromboembolismo venoso (TEV) en pacientes con indicación de tromboprofilaxis hospitalizados en los servicios de medicina de dos hospitales generales de Lima

\begin{tabular}{|c|c|c|c|c|c|c|c|c|c|c|c|c|}
\hline \multirow[b]{2}{*}{$\begin{array}{l}\text { Factores de } \\
\text { riesgo, } \mathbf{n}(\%)\end{array}$} & \multicolumn{3}{|c|}{ Medición basal } & \multirow[b]{2}{*}{$\begin{array}{c}\text { Valor } \\
p\end{array}$} & \multicolumn{3}{|c|}{ Primera medición } & \multirow[b]{2}{*}{$\begin{array}{c}\text { Valor } \\
p\end{array}$} & \multicolumn{3}{|c|}{ Segunda medición } & \multirow[b]{2}{*}{$\begin{array}{c}\text { Valor } \\
p\end{array}$} \\
\hline & $\begin{array}{l}\text { Total } \\
(n=55)\end{array}$ & $\begin{array}{l}\text { Control } \\
(n=32)\end{array}$ & $\begin{array}{l}\text { Intervenido } \\
\quad(n=23)\end{array}$ & & $\begin{array}{l}\text { Total } \\
(n=53)\end{array}$ & $\begin{array}{l}\text { Control } \\
(n=24)\end{array}$ & $\begin{array}{c}\text { Intervenido } \\
\quad(n=29)\end{array}$ & & $\begin{array}{l}\text { Total } \\
(n=68)\end{array}$ & $\begin{array}{c}\text { Control } \\
(n=33)\end{array}$ & $\begin{array}{l}\text { Intervenido } \\
\quad(n=35)\end{array}$ & \\
\hline Edad $\geq 70$ & $33(60,0 \%)$ & $18(56,3 \%)$ & $15(65,2 \%)$ & 0,503 & $18(33,9 \%)$ & $7(29,2 \%)$ & $11(37,9 \%)$ & 0,502 & $34(50,0 \%)$ & $13(39,4 \%)$ & $21(60,0 \%)$ & 0,089 \\
\hline Neoplasia & $10(18,2 \%)$ & $7(21,9 \%)$ & $3(13,0 \%)$ & 0,402 & $17(32,1 \%)$ & $4(16,7 \%)$ & $13(44,8 \%)$ & 0,029 & $13(19,1 \%)$ & $5(15,2 \%)$ & $8(22,9 \%)$ & 0,419 \\
\hline TEV previo & 0 & 0 & 0 & - & $3(5,7 \%)$ & 0 & $3(10,3 \%)$ & 0,105 & $4(5,9 \%)$ & 0 & $4(11,4 \%)$ & 0,045 \\
\hline Movilización & $50(90,9 \%)$ & $29(90,6 \%)$ & $21(91,3 \%)$ & 0,931 & $41(77,4 \%)$ & $22(91,7 \%)$ & $19(65,5 \%)$ & 0,024 & $58(85,3 \%)$ & $30(90,9 \%)$ & $28(80,0 \%)$ & 0,204 \\
\hline Trombofilia & 0 & 0 & 0 & - & 0 & 0 & 0 & - & 0 & 0 & 0 & - \\
\hline $\begin{array}{l}\text { Trauma y/o } \\
\text { cirugía }\end{array}$ & $6(10,9 \%)$ & $5(15,6 \%)$ & $1(4,4 \%)$ & 0,186 & $6(11,3 \%)$ & $2(8,3 \%)$ & $4(13,8 \%)$ & 0,532 & $6(8,8 \%)$ & $4(12,1 \%)$ & $2(5,7 \%)$ & 0,352 \\
\hline $\begin{array}{l}\text { Falla cardiaca } \\
\text { y/o respiratoria }\end{array}$ & $17(30,9 \%)$ & $10(31,3 \%)$ & $7(30,4 \%)$ & 0,949 & $13(24,5 \%)$ & $6(25,0 \%)$ & $7(24,1 \%)$ & 0,942 & $12(17,7 \%)$ & $6(18,2 \%)$ & $6(17,1 \%)$ & 0,911 \\
\hline IMA o ACV & $3(5,5 \%)$ & $1(3,1 \%)$ & $2(8,7 \%)$ & 0,370 & $4(7,6 \%)$ & $4(16,7 \%)$ & 0 & 0,022 & $10(14,7 \%)$ & $4(12,1 \%)$ & $6(17,1 \%)$ & 0,559 \\
\hline $\begin{array}{l}\text { Infección y/o } \\
\text { reumatológico }\end{array}$ & $22(40,0 \%)$ & $14(43,8 \%)$ & $8(34,8 \%)$ & 0,503 & $30(56,6 \%)$ & $12(50,0 \%)$ & $18(62,1 \%)$ & 0,378 & $37(54,4 \%)$ & $24(72,7 \%)$ & $13(37,1 \%)$ & 0,003 \\
\hline Obesidad & $8(14,6 \%)$ & $4(12,5 \%)$ & $4(17,4 \%)$ & 0,612 & $8(15,1 \%)$ & $7(29,2 \%)$ & $1(3,5 \%)$ & 0,009 & $9(13,2 \%)$ & $6(18,2 \%)$ & $3(8,6 \%)$ & 0,242 \\
\hline $\begin{array}{l}\text { Terapia } \\
\text { hormonal }\end{array}$ & $1(1,8 \%)$ & 0 & $1(4,4 \%)$ & 0,234 & 0 & 0 & 0 & - & $8(11,8 \%)$ & $4(12,1 \%)$ & $4(11,4 \%)$ & 0,929 \\
\hline
\end{tabular}


Tabla 4. Frecuencia de pacientes hospitalizados con prescripción acorde a las guías de práctica clínica en los servicios de Medicina en dos hospitales generales de Lima

\begin{tabular}{|c|c|c|c|c|c|c|c|c|c|}
\hline \multirow{2}{*}{$\begin{array}{l}\text { Prescripción } \\
\text { acorde a guías } \\
\text { de práctica } \\
\text { clínica }\end{array}$} & \multicolumn{3}{|c|}{ Medición basal } & \multicolumn{3}{|c|}{ Primera medición } & \multicolumn{3}{|c|}{ Segunda medición } \\
\hline & $\begin{array}{l}\text { Control } \\
(n=60)\end{array}$ & $\begin{array}{c}\text { Intervenido } \\
\quad(n=62)\end{array}$ & $\begin{array}{c}\text { Valor } \\
p\end{array}$ & $\begin{array}{l}\text { Control } \\
(n=55)\end{array}$ & $\begin{array}{c}\text { Intervenido } \\
\quad(n=75)\end{array}$ & $\underset{p}{\text { Valor }}$ & $\begin{array}{l}\text { Control } \\
(n=52)\end{array}$ & $\begin{array}{l}\text { Intervenido } \\
\quad(n=90)\end{array}$ & $\underset{p}{\text { Valor }}$ \\
\hline Sí & $34(56,7 \%)$ & $40(64,5 \%)$ & 0,483 & $35(63,6 \%)$ & $47(62,7 \%)$ & 0,910 & $29(55,8 \%)$ & $49(54,4 \%)$ & 0,879 \\
\hline No & $26(43,3 \%)$ & $22(35,5 \%)$ & & $20(36,4 \%)$ & $28(37,3 \%)$ & & $23(44,2 \%)$ & $41(45,6 \%)$ & \\
\hline
\end{tabular}

( $n=49)$. Por otro lado, en el hospital control fue de $56,7 \%$ $(n=34) ; 63,6 \%(n=35)$, y 55,8\% $(n=29)$, respectivamente; siendo sus valores de $p=0,483 ; p=0,910 ; p=0,879$ para cada medición (Tabla 4, Figura 1).

En el hospital intervenido, en la medición basal, el $10,0 \%(n=4)$ de hojas de terapéutica con prescripción acorde a GPC en pacientes con indicación aumentó a $25,5 \%(n=12)$ en la primera medición, para disminuir a $10,2 \%(n=5)$ en la última. Por otro lado, inicialmente en el hospital control, la prescripción acorde a GPC en pacientes con indicación se presentó en 38,2\% ( $n=13)$ de las hojas de terapéutica, obteniéndose un $44,8 \%$ $(n=13)$ en la última medición.

En cuanto a los pacientes sin indicación, en la medición basal del hospital intervenido, el 90,0\% ( $n=36)$ no tenía prescripción en las hojas de terapéutica, lo cual disminuyó a $74,5 \%(n=35)$ en la primera medición para luego aumentar a $89,8 \%(n=44)$ en la última; mientras que en el hospital control esta fue de $61,7 \% \quad(n=21)$, $80,0 \%(n=28)$ y $55,2 \% \quad(n=16)$ en la medición basal, primera y segunda, respectivamente.

En cuanto a la prescripción no acorde a GPC, en el hospital intervenido fue del 35,5\% ( $n=22)$ en la medición basal. De esto, el porcentaje de hojas terapéuticas con subuso de tromboprofilaxis significó el 86,4\% ( $n=19)$ y el sobreuso $13,6 \%(n=3)$. En la última medición, el sobreuso incrementó a $26,8 \%(n=11)$; mientras que el subuso disminuyó a $73,2 \%(n=30)$. Por otro lado, en el hospital control, el porcentaje de hojas de terapéutica con subuso incrementó de un $73,1 \%(n=19)$ a un $86,9 \%$ $(n=20)$; mientras que el sobreuso disminuyó de un $26,9 \%(n=7)$ a $13,0 \%(n=3)$ (Figura 2$)$.

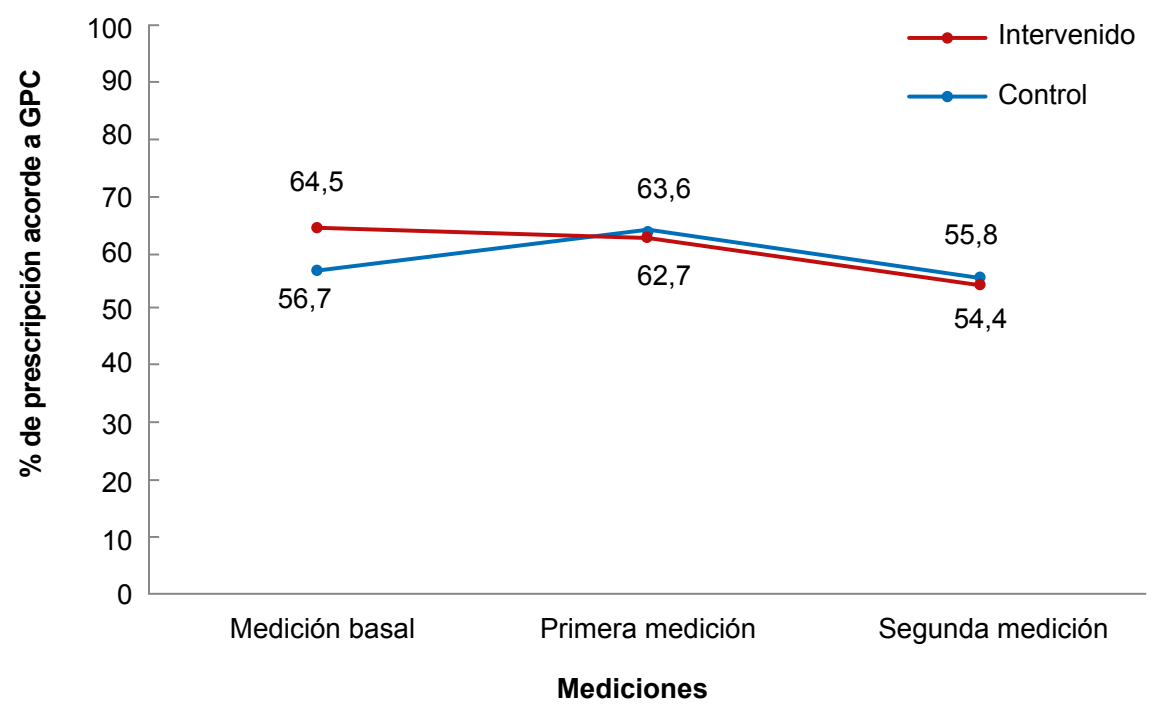

Figura 1. Prescripción de tromboprofilaxis acorde a guías de práctica clínica (GPC) en pacientes hospitalizados de los servicios de Medicina de dos hospitales docentes de Lima

IMA: Infarto de miocardío agudo, ACV: Accidente cerebro vascular 


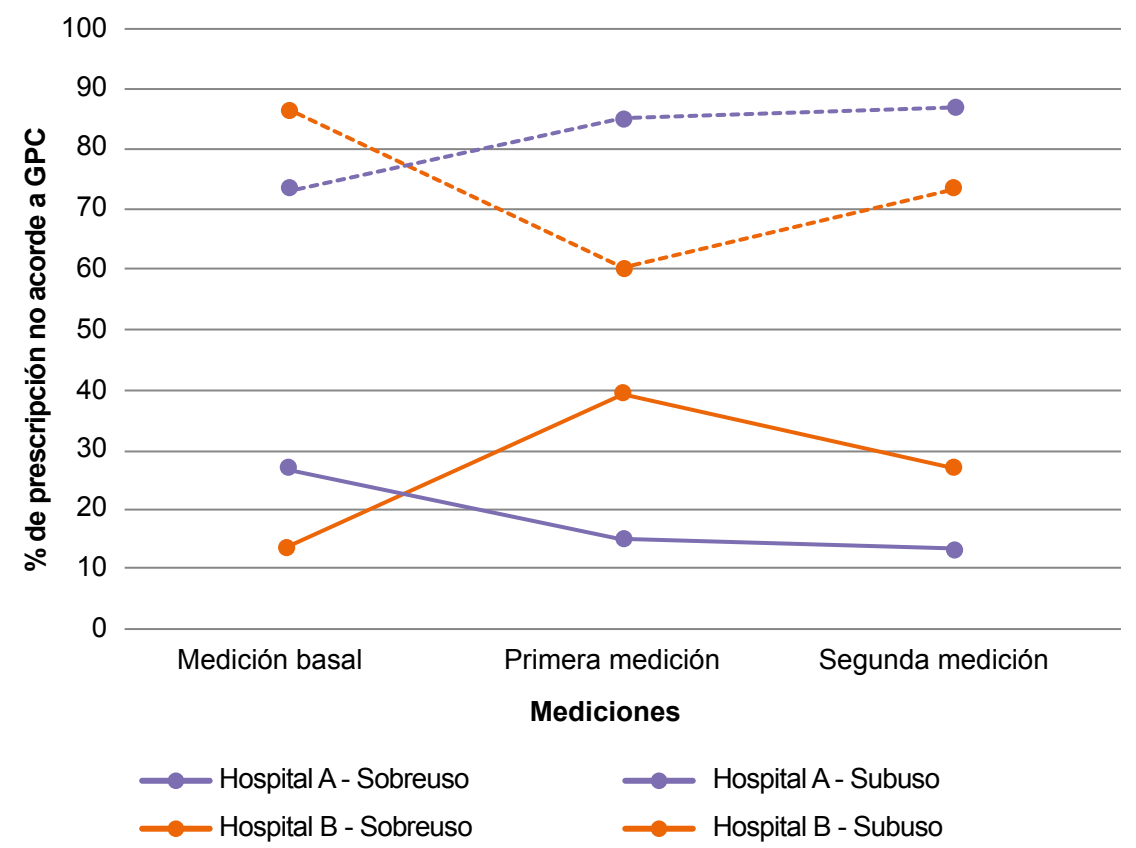

Figura 2. Prescripción de tromboprofilaxis no acorde a las guías de práctica clínica (GPC) en pacientes hospitalizados de los servicios de medicina de dos hospitales docentes de Lima

\section{DISCUSIÓN}

La aplicación de alertas electrónicas a través de mensajería de texto y correos electrónicos, aplicados a médicos residentes de un hospital general, no tuvo impacto en el incremento de la prescripción de tromboprofilaxis acorde a GPC a pacientes hospitalizados en salas de medicina interna.

En el campo de las terapias preventivas se conoce que la tromboprofilaxis es efectiva para reducir la morbimortalidad en los pacientes hospitalizados con riesgo para TEV. En los hospitales evaluados, la medición preintervención mostró que del total de pacientes con indicación de tromboprofilaxis solo alrededor del 60\% tenían prescripción acorde a GPC. A diferencia de otros estudios que, habitualmente, definen la variable "prescripción acorde a GPC" como la prescripción en pacientes con indicación, nuestro estudio incluyó también a los pacientes sin indicación que no tenían prescripción. Si se toma en cuenta la definición habitual, los resultados basales fueron de $38,2 \%$ en el hospital control y $10,0 \%$ en el intervenido, comparables con lo reportado en el estudio ENDORSE 2008, el cual incluyó a servicios de medicina de hospitales de 32 países, donde la tromboprofilaxis fue prescrita acorde a GPC solo en el $39,5 \%$ de pacientes con indicación ${ }^{(12,21-23)}$.

Diversos estudios en servicios de salud muestran a las TIC, y específicamente a la mensajería de texto, como el medio preferido por los médicos residentes para el intercambio de información sobre el cuidado del paciente ${ }^{(24,25)}$. Para mejorar la adherencia a la aplicación de las GPC se han obtenido resultados favorables con la aplicación de TIC, además del uso de métodos tradicionales (difusión presencial, sesiones educativas) ${ }^{(26)}$. En un estudio en el que se comparó la enseñanza tradicional (libros, folletos) con el envío de información a través de mensajes de texto, este último resultó tener mayor tasa de aprendizaje entre los médicos residentes de un servicio de ginecoobstetricia ${ }^{(27)}$. De igual manera, la intervención basada en el envío de mensajes de texto para mejorar la adherencia al tratamiento en pacientes con enfermedades crónicas ha sido ampliamente estudiada y han logrado éxito ${ }^{(28-30)}$.

Existen diversos factores que pudieron influenciar en que el resultado de nuestro estudio no haya sido el esperado. La apertura y revisión de los mensajes no pudo ser verificado debido a que no se contó con un sistema de retroalimentación, siendo esto un gran limitante para medir el impacto real de la intervención. Además, pese a que los participantes seleccionaron la presentación de los mensajes de texto y su frecuencia de envío a través de un grupo focal, es probable que su elección no haya sido la más apropiada y atractiva. Por otra parte, la mayoría de estudios sobre recordatorios con resultados favorables fueron desarrollados en países con mayor aplicación de TIC en el sistema de salud, donde utilizan historias clínicas electrónicas. Esto último permite el uso de recordatorios a través de ventanas emergentes (pop-ups), alertas electrónicas, los cuales son generados 
automáticamente por el software para evaluar el riesgo del clínico del paciente o el uso de cierta droga al momento de indicar la prescripción ${ }^{(24,31,32)}$.

En nuestro medio existe baja adherencia a la aplicación de GPC en la prescripción de pacientes hospitalizados. En un estudio previo realizado en estos dos hospitales, se observó un elevado sobreuso de inhibidores de bomba de protones en pacientes hospitalizados, específicamente porque los médicos consideran indicaciones de uso que no están basadas en recomendaciones por las GPC ${ }^{(33)}$. Un estudio latinoamericano expone que la primera causa para la baja adherencia a la guía institucional radica en el olvido de la prescripción, pues el médico no tiene incorporado dentro de sus hábitos regulares la idea de tromboprofilaxis, y muchas veces lo prescribe, pero a dosis inadecuadas, como ocurrió en nuestro estudio ${ }^{(34)}$. Estas situaciones pueden deberse a que, generalmente, no se ha realizado el proceso de implementación de GPC para su uso en los servicios de hospitalización.

Luego de la intervención se evidenció que la disminución en la prescripción acorde a GPC se debió a un aumento en el sobreuso. Esto sugeriría que los mensajes de texto enviados hayan tenido un tono imperativo que produzca en los médicos residentes la sensación de una orden de prescribir tromboprofilaxis en lugar de ser un recordatorio de cuando se recomienda su aplicación.

Entre las limitaciones del estudio tenemos que la GPC utilizada no ha sido adaptada ni implementada en los hospitales del Ministerio de Salud del Perú, sin embargo los hospitales evaluados son sedes docentes para la formación de médicos residentes y la GPC utilizada es la se imparte en su clases y rondas clínicas durante el programa de especialización según pudimos verificar. No realizamos una valoración del nivel de conocimiento sobre tromboprofilaxis previa a la intervención; según la pirámide de aprendizaje de Miller, es necesario realizar una valoración previa sobre el nivel de conocimiento o aprendizaje antes de evaluar el desempeño real de la práctica profesional ${ }^{(35)}$. Esto es especialmente relevante cuando se evalúa la tromboprofilaxis no farmacológica, ya que no sabemos que no la prescriben por desconocimiento o falta de disponibilidad en su servicio.
Entre las fortalezas de este trabajo tenemos que es un primer intento en la aplicación de TIC como recordatorios que sirvan como herramienta educativa en el campo de la medicina de nuestro país, existen muy pocos trabajos publicados en la literatura médica para el caso de tromboprofilaxis. Las TIC no solo pueden ser útiles para la prescripción de fármacos por los médicos residentes, sino también para abordar otros problemas vigentes que tienen los médicos residentes durante su especialización, el brindar cursos presenciales tiene la limitación de que se cuenta con pocas horas disponibles ya que generalmente existe sobrecarga de labores asistenciales.

Como conclusión, tenemos que en este estudio no tuvo impacto la aplicación de una intervención basada en el uso de mensajes de texto y correos electrónicos como recordatorios para la prescripción de tromboprofilaxis acordes a GPC, en médicos residentes de primer año de un hospital general. Recomendamos que en posteriores intervenciones se utilicen estrategias múltiples simultáneas con componentes más activos, en los que sea posible verificar el nivel de conocimiento y la aplicación de todo el personal involucrado en la prescripción.

Agradecimientos: a la doctora María Lazo Porras por su colaboración en el análisis estadístico del presente estudio. A los Médicos Internistas Pedro Ortiz Saavedra (HAL) y Antonio Hernández Sánchez $(\mathrm{HCH})$ por su valiosa colaboración.

Contribuciones de autoría: MÁGT, KGT y RMDZ han participado en la concepción del artículo; recolección de resultados, análisis e interpretación de datos, redacción del artículo y su revisión crítica. Asimismo, la Dra. María de los Ángeles Lazo Porras participó en el análisis de datos. Además, RTA ha participado en la concepción del artículo, análisis e interpretación de los datos, su revisión crítica y aprobación de la versión final, mientras que WML brindó asesoría estadística.

Fuentes de financiamiento: este trabajo de investigación fue financiado por la Beca Anual de Medicina "Francisco Tejada y Semiramis Reátegui"- Facultad de Medicina Alberto Hurtado. Universidad Peruana Cayetano Heredia

Conflictos de interés: los autores declaran no tener conflictos de interés en la publicación de este artículo.

Anexos disponibles en: http://www.rpmesp.ins.gob.pe/index. php/rpmesp/article/view/2372/2268

\section{REFERENCIAS BIBLIOGRÁFICAS}

1. Zhan C, Miller MR. Excess length of stay, charges, and mortality attributable to medical injuries during hospitalization. JAMA. 2003;290(14):1868-74. doi:10.1001/ jama.290.14.1868.

2. Kahn S, Lim W, Dunn A, Cushman M, Dentali F, Akl EA, et al. Prevention of VTE in Nonsurgical Patients Antithrombotic Therapy and
Prevention of Thrombosis, 9th ed: American College of Chest Physicians Evidence-Based Clinical Practice Guidelines. Chest. 2012;141(2) (Suppl):e195S-e226S. doi: 10.1378/ chest.11-2296.

3. Shojania KG, Duncan BW, McDonald KM, Wachter RM, eds. Making Health Care Safer. A Critical Analysis of Patient Safety Practices. Evidence
Report/Technology Assessment, No. 43 [internet]. Rockville, MD: Agency for Healthcare Research and Quality/ U.S. Department of Health and Human Services; 2001 [citado el el 27 de abril de 2016]. Disponible en: http:// archive.ahrq.gov/clinic/ptsafety/pdf/ ptsafety.pdf

4. Aujesky D, Roy PM, Le Manach CP, Verschuren F, Meyer G, Obrosky DS, 
et al. Validation of a model to predict adverse outcomes in patients with pulmonary embolism. Eur Heart J. 2006(4);27:476-81. doi: http://dx.doi. org/10.1093/eurheartj/ehi588

5. Sullivan SD, Kahn SR, Davidson BL, Borris L, Bossuyt P, Raskob G. Measuring the outcomes and pharmacoeconomic consequences of venous thromboembolism prophylaxis in major orthopaedic surgery. Pharmacoeconomics. 2003;21(7):477-96.

6. Caprini JA, Botteman MF, Stephens JM, Nadipelli V, Ewing MM, Brandt $S$, et al. Economic burden of long-term complications of deep vein thrombosis after total hip replacement surgery in the United States. Value Health. 2003;6(1):59-74.

7. Avorn J, Winkelmayer WC. Comparing the costs, risks, and benefits of competing strategies for the primary prevention of venous thromboembolism. Circulation. 2004;110(24 Suppl 1):IV25-IV32.

8. Anderson FA Jr, Spencer FA. Risk Factors for Venous Thromboembolism. Circulation. 2003;107(23 Suppl 1):I9-16. doi: 10.1161/01. CIR.0000078469.07362.E6

9. Heit J, O'Fallon WM, Petterson TM, Lohse CM, Silverstein MD, Mohr DN, et al. Relative Impact of Risk Factors for Deep Vein Thrombosis and Pulmonary Embolism: a population-based study. Arch Intern Med. 2002;162(11):12458. doi:10.1001/archinte.162.11.1245.

10. Rosendaal FR. Venous thrombosis: a multicausal disease. Lancet. 1999;353(9159):1167-73. doi: http://dx.doi.org/10.1016/S01406736(98)10266-0

11. Anderson FA Jr, Wheeler HB, Goldberg RJ, Hosmer DW, Forcier A. The prevalence of risk factors for venous thromboembolism among hospital patients. Arch Intern Med. 1992;152(8):1660-4. doi:10.1001/ archinte.1992.00400200092017.

12. Cohen AT, Tapson VF, Bergmann JF, Goldhaber SZ, Kakkar AK, Deslandes B, et al. Venous thromboembolism risk and prophylaxisin the acute hospital care setting (ENDORSE study): a multinational cross-sectional study. Lancet. 2008;371(9610):387-94. doi: 10.1016/S0140-6736(08)60202-0.

13. Khalili H, Dashti-Khavidaki $S$, Talasaz AH, Najmedin F, Hosseinpoor R. Anticoagulant utilization evaluation in a teaching hospital: a prospective study. J Pharm Pract. 2010;23(6):579-84. doi: $10.1177 / 0897190010372808$.
14. Tooher R, Middleton P, Pham C, Fitridge R, Rowe S, Babidge W, et al. A Systematic Review of Strategies to Improve Prophylaxis for Venous Thromboembolism in Hospitals. Ann Surg. 2005;241(3):397-415. doi: 10.1097/01.sla.0000154120.96169.99.

15. Schunemann HJ, Cook D, Grimshaw J, Liberati A, Heffner J, Tapson V, et al. Antithrombotic and thrombolytic therapy: from evidence to application: the Seventh ACCP Conference on Antithrombotic and Thrombolytic Therapy. Chest. 2004;126(3 Suppl):688S-696S.

16. Agámez $S$, Aldana $M$, Barreto V, Santana A, Caballero-Uribe CV. Aplicación de nuevas tecnologías de la información en la enseñanza de la medicina. Salud Uninorte Barranquilla (Col). 2009;25(1):150-71.

17. Mosen D, Elliott CG, Egger MJ, Mundorff M, Hopkins J, Patterson R, et al. The effect of a computerized reminder system on the prevention of postoperative venous thromboembolism. Chest. 2004; 125(5):1635-41. doi:10.1378/ chest.125.5.1635

18. Kucher N, Koo S, Quiroz R, Cooper JM, Paterno MD, Soukonnikov B, et al. Electronic alerts to prevent venous thromboembolism among hospitalized patients. N Engl J Med. 2005;352(10):969-77. doi: 10.1056/ NEJMoa041533

19. Ministerio de Transporte y Comunicaciones (MTC). Líneas en servicio y densidad en la telefonía fija y móvil. 1993-2011 [internet]. Lima: MTC; c2016 [citado 15 abril 2012]. Disponible en: http://www.mtc.gob. pe/estadisticas/archivos/

20. Barbar S, Noventa F, Rossetto V, Ferrari A, Brandolin B, Perlati M, et al. A risk assessment model for the identification of hospitalized medical patients at risk for venous thromboembolism: the Padua Prediction Score. J Thromb Haemost. 2010;8(11):2450-7. doi: 10.1111/j.1538-7836.2010.04044.x.

21. Melero MJ, Pagotto VL, Mazzei JA. Tromboprofilaxis en pacientes no quirúrgicos internados en un hospital general. Medicina (B Aires). 2012;72(5):361-6.

22. Kerbauy MN, de Moraes FY, Kerbauy LN, Conterno Lde O, El-Fakhouri S. Venous thromboprophylaxis in medical patients: an application review. Rev Assoc Med Bras. 2013;59(3):258-64. doi: 10.1016/j.ramb.2012.11.008.
23. Ageno W, Squizzato A, Ambrosini F, Dentali F, Marchesi C, Mera V, et al. Thrombosis prophylaxis in medical patients: a retrospective review of clinical practice patterns. Haematologica. 2002;87(7):746-50.

24. Shah DR, Galante JM, Bold RJ, Canter RJ, Martinez SR. Text Messaging Among Residents and Faculty in a University General Surgery Residency Program: Prevalence, Purpose, and Patient Care. J Surg Educ. 2013;70(6):826-34. doi: 10.1016/j.jsurg.2012.05.003.

25. Vásquez-Silva L, Ticse R, AlfaroCarballido L, Guerra-Castañon F. Acceso, uso y preferencias de las tecnologías de información y comunicación por médicos de un hospital general del Perú. Rev Peru Med Exp Salud Publica. 2015;32(2):289-93.

26. Kahn SR, Morrison DR, Cohen JM, Emed J, Tagalakis V, Roussin A, et al. Interventions for implementation of thromboprophylaxis in hospitalized medical and surgical patients at risk for venous thromboembolism. Cochrane Database Syst Rev. 2013;7: CD008201. doi: 10.1002/14651858. CD008201.pub2.

27. Alipour S, Moini A, Jafari-Adii S, Gharaie N, Mansouri K. Comparison of teaching about breast cancer via mobile or traditional learning methods in gynecology residents. Asian Pac J Cancer Prev. 2012;13(9):4593-5.

28. Kurumop SF, Bullen C, Whittaker R, Betuela I, Hetzel MW, Pulford J. Improving health worker adherence to malaria treatment guidelines in Papua New Guinea: feasibility and acceptability of a text message reminder service. PLoS One. 2013;8(10):e76578. doi: 10.1371/journal.pone.0076578.

29. Sahu M, Grover A, Joshi A. Role of mobile phone technology in health education in Asian and African countries: a systematic review. Int J Electron Healthc. 2014;7(4):269-86. doi: 10.1504/IJEH.2014.064327.

30. Vervloet M, Linn AJ, van Weert JC, de Bakker DH, Bouvy ML, van Dijk L. The effectiveness of interventions using electronic reminders to improve adherence to chronic medication: a systematic review of the literature. J Am Med Inform Assoc. 2012;19(5):696-704. doi: 10.1136/ amiajnl-2011-000748.

31. Sliwka D, Fang MC. Venous Thromboembolism Prophylaxis in the United States: Still Room for Improvement. J Gen Intern Med. 
2010;25(6):484-6. doi: 10.1007/ s11606-010-1350-9.

32. Al-Hameed F, Al-Dorzi HM, AboelnazerE. The effect of a continuing medical education program on Venous thromboembolism prophylaxis utilization and mortality in a tertiarycare hospital. Thromb J. 2014;12:9. doi: 10.1186/1477-9560-12-9.

33. Bustamante Robles KY, Ticse Aguirre R, Cánepa Rondo IF, Costta Herrera CG, Vasquez Kunze S, Soto Arquiñigo $\mathrm{L}$, et al. Frecuencia de la prescripción de los inhibidores de bomba de protones basada en guías de práctica clínica en pacientes hospitalizados en dos hospitales docentes de Lima-Perú. Rev Gastroenterol Perú. 2012;32(1):44-9.

34. Velásquez JC, Herrán S, Mera I. Uso de tromboprofilaxis en el paciente hospitalizado en la Clínica del Country. Un análisis de la adherencia a la guía institucional. Acta Med Colomb. 2010;35(1):1-14.

35. Wass V, Van der Vleuten C, Shatzer J, Jones R. Assessment of clinical competence. Lancet. 2001;357(9260):945-9. doi http://dx.doi.org/10.1016/S01406736(00)04221-5.

Correspondencia: Ray Willy Ticse Aguirre

Dirección: Servicio de Endocrinología - Hospital Nacional Cayetano Heredia - Av. Honorio Delgado 264 - San Martín de Porres

Teléfono: 998009724

Correo electrónico: ray.ticse@upch.pe

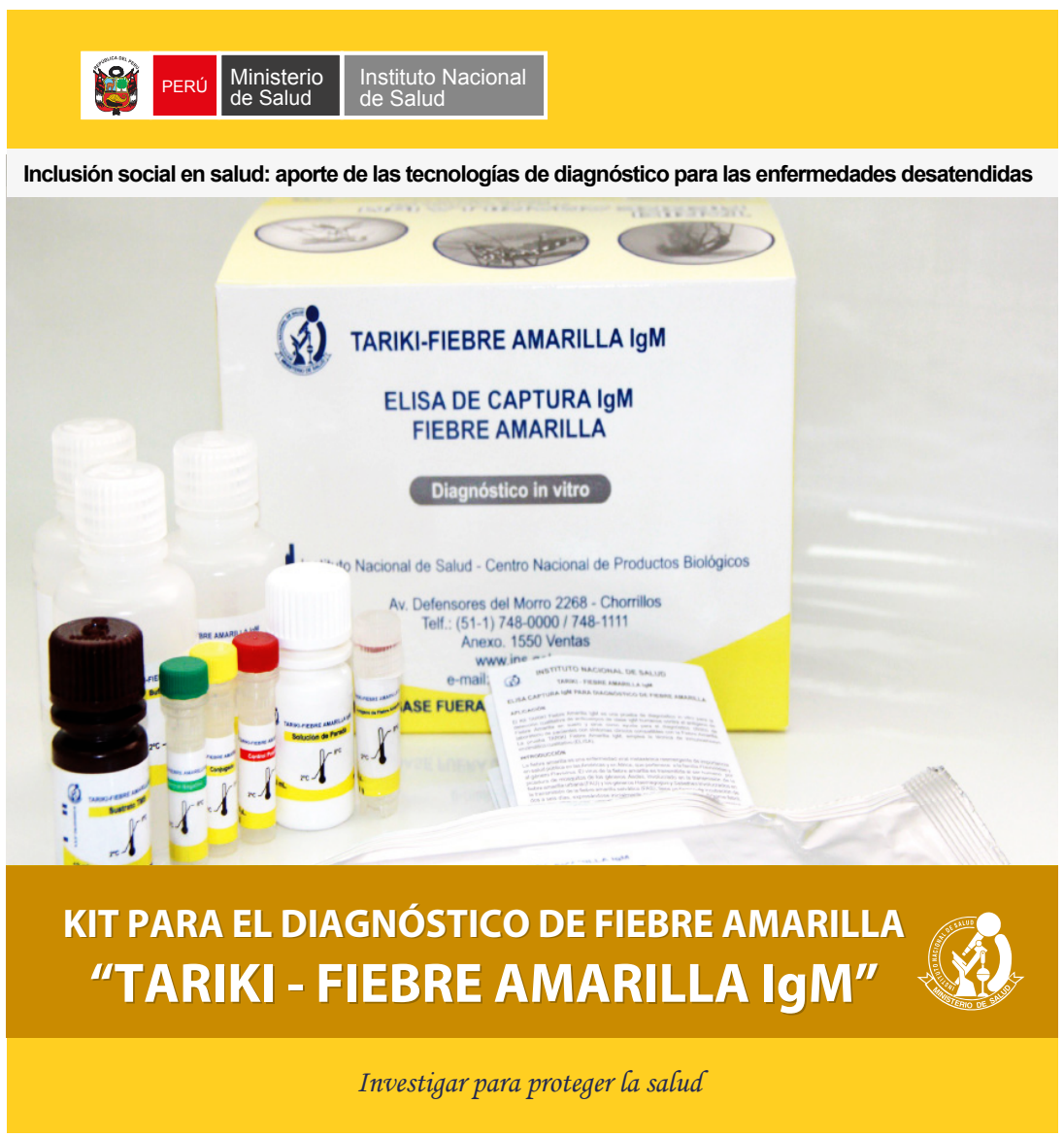




\section{ANEXOS}

Anexo 1. Condiciones que no requieren tromboprofilaxis

Cirugía general de bajo riesgo y sin factores de riesgo adicionales (sólo deambulación precoz)

Cirugía vascular que no tienen otros factores de riesgo (sólo deambulación precoz)

Cirugía ginecológica de bajo riesgo sin factores de riesgo adicionales (sólo deambulación precoz)

Cirugía ginecológica laparoscópica (sólo deambulación precoz)

Procedimientos transuretrales u otros de bajo riesgo (sólo deambulación precoz)

Procedimientos laparoscópicos y sin factores de riesgo (sólo deambulación precoz)

Pacientes sometidos a artroscopía de rodilla sin otros factores de riesgo (movilización precoz)

Lesiones aisladas de extremidades inferiores, distales a la rodilla

Pacientes con cáncer y CVC permanente

Pacientes con cáncer que reciben quimioterapia o terapia hormonal

Pacientes con alto riesgo de sangrado (tromboprofilaxis mecánica)*

*Tiene contraindicación para tromboprofilaxis farmacológica, por lo que se recomienda mecánica.

Anexo 2. Lista de mensajes enviados

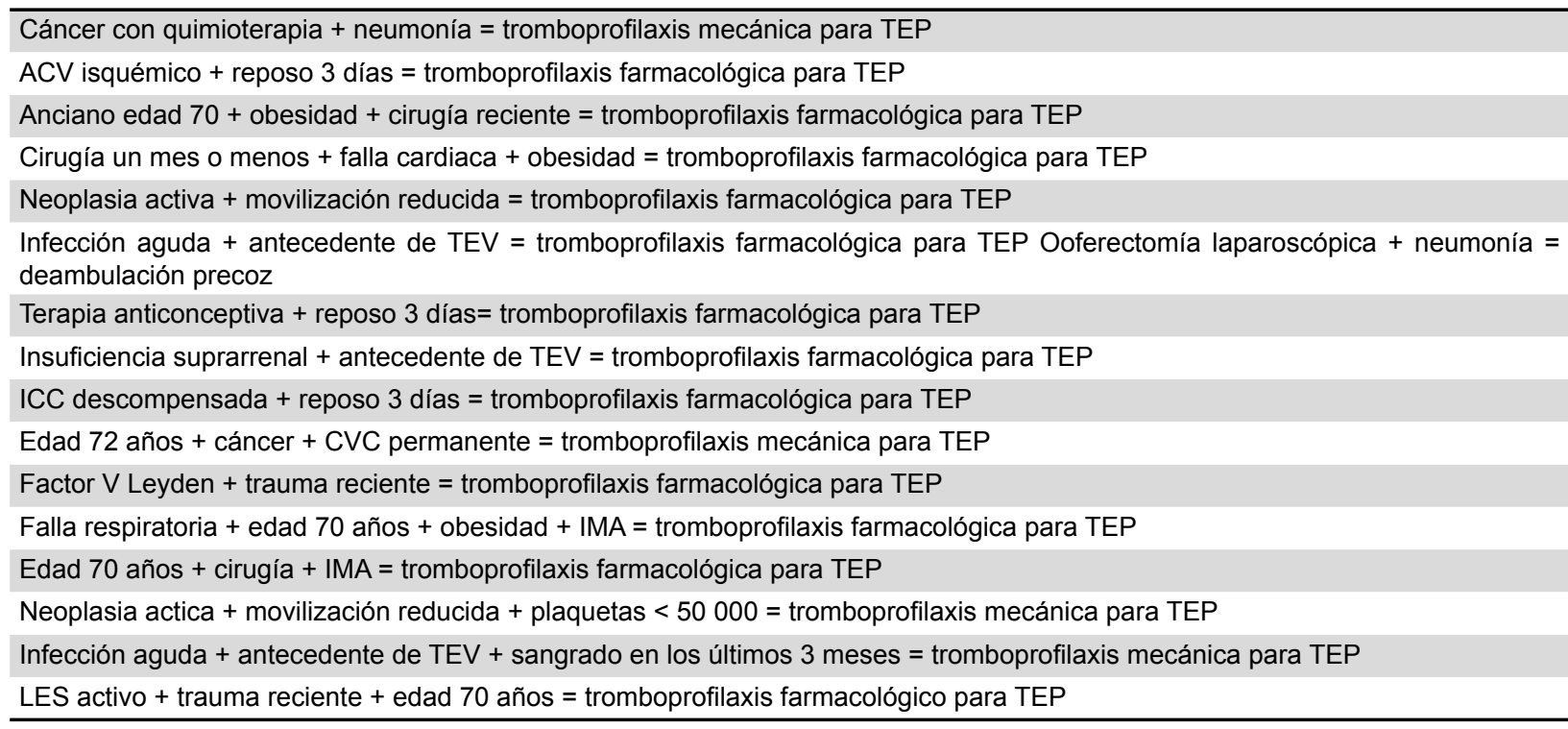

